\title{
FROM HERMITEAN CLIFFORD ANALYSIS TO SUBELLEPITIC DIRAC OPERATORS ON ODD DIMENSIONAL SPHERES AND OTHER CR MANIFOLDS
}

\author{
P. CEREJEIRAS, U. KÄHLER, AND J. RYAN
}

\begin{abstract}
We show that the two Dirac operators arising in Hermitian Clifford analysis are identical to standard differential operators arising in several complex variables. We also show that the maximal subgroup that preserves these operators are generated by translations, dilations and actions of the unitary n-group. So the operators are not invariant under Kelvin inversion. We also show that the Dirac operators constructed via two by two matrices in Hermitian Clifford analysis correspond to standard Dirac operators in euclidean space. In order to develop Hermitian Clifford analysis in a different direction we introduce a sub elliptic Dirac operator acting on sections of a bundle over odd dimensional spheres. The particular case of the three sphere is examined in detail. We conclude by indicating how this construction could extend to other CR manifolds.
\end{abstract}

Keywords: Kohn Laplacian; Kohn Dirac operator; CR manifolds;

MSC(2010): primary: 30G35 ; secondary: 53C27

\section{INTRODUCTION}

Clifford analysis started as an attempt to generalize one variable complex analysis to n-dimensional euclidean space. It has since evolved into a study of the analyst, geometry and applications of Dirac operators over euclidean space, spheres, real projective spaces, conformally flat spin manifolds and spin manifolds with applications to representation theory arising from mathematical physics, classical harmonic analysis and many other topics.

In recent years a topic referred to as Hermitian Clifford analysis has attracted some attention. See for instance $[2,3,4]$. It is developed initially over a complexification of even dimensional Euclidean space. An almost complex structure is introduced and two associated projection operators are applied to this complex vector space. This splits this space into two n-dimensional complex spaces. When these operators are applied to the euclidean Dirac operator it splits into two differential operators. Here we show that these operators are respectively the d-bar operator and its dual as arising in several complex variables. See for instance $[5,6]$.

The Euclidean Dirac operator is a conformally invariant operator. In particular it is invariant under Kelvin inversion. We show that these Hermitian Dirac operators have a narrower range of invariance. We show they are no longer invariant under Kelvin inversion. Their maximal invariance group is generated by translation, dilation and a subgroup of $S O(2 n)$ isomorphic to $U(n)$.

We also show via lemma given in [1] that the Dirac operator constructed via two by two matrices is in fact a standard Dirac operator over Euclidean space. In order to try and develop fresh ideas for this topic we transfer to odd dimensional spheres and make use of their CR structure to introduce a subelliptic Dirac operator acting on sections of a bundle defined over the sphere. Each fiber is isomorphic to a Clifford algebra generated from the CR structure of the sphere. The square of this Dirac operator gives the Kohn Laplacian on the sphere. The Hopf vibration is used to illustrate this scenario in more detail over $\mathbb{S}^{3}$. We conclude by illustrating how this construction might carry over to other CR manifolds. 


\section{Preliminaries on Hermitean Clifford Analysis}

In Hermitian Clifford analysis one starts with the standard Dirac operator

$$
D=\sum_{j=1}^{n} e_{j} \frac{\partial}{\partial x_{j}}
$$

in the Euclidean space $\mathbb{R}^{m}$. Here

$$
e_{i} e_{j}+e_{j} e_{i}=-2 \delta_{i j}
$$

under Clifford algebra multiplication.

So we consider the real $2^{m}$ dimensional Clifford algebra $\mathcal{C} \ell_{m,-}$ with $\mathbb{R}^{m} \subset \mathcal{C} \ell_{m,-}$ and $x^{2}=-\|x\|^{2}$, for all each $x \in \mathbb{R}^{m}$.

One now introduces an almost complex structure on $\mathbb{R}^{m}$. This is a matrix $J \in S O(m)$ with $J^{2}=-I$. As $J \in S O(m)$ this forces $m$ to be even. So $m=2 n$. For instances, $J=\left(\begin{array}{cc}0 & -I \\ I & 0\end{array}\right)$. This is the choice of $J$ that we will use here. We now complexify $\mathbb{R}^{2 n}$ to obtain $\mathbb{C}^{2 n}$ and we complexify $\mathcal{C}_{2 n,-}$ to obtain the complex Clifford algebra $\mathrm{Cl}_{2 n}(\mathbb{C})$. Following $[5,6]$ we now have the projection operators $\frac{1}{2}(I \pm i J)$. They act on $\mathbb{C}^{2 n}$ and split it into two complex spaces $W^{+} \oplus W^{-}$each of complex dimension $n$. So

$$
W^{ \pm}=\frac{1}{2}(I \pm i J) \mathbb{C}^{2 n}
$$

In particular, $\frac{1}{2}(I \pm i J) e_{j}=\frac{1}{2}\left(e_{j} \pm i e_{j+n}\right)$, for $j=1, \ldots, n$, and it equals $\frac{1}{2}\left(e_{j} \mp i e_{j-n}\right)$, for $j=n+1, \ldots, 2 n$. Note $\frac{1}{2}\left(e_{j} \mp i e_{j-n}=\frac{1}{2} i\left(e_{k} \pm i e_{k+n}\right)\right.$ for $j=n+1, \cdots, 2 n$ and $k=j-n$.

We denote $\frac{1}{2}\left(e_{k} \pm i e_{k+n}\right)$ by $\mathfrak{f}_{k}^{ \pm}$respectively, with $k=1, \cdots, n$. The elements $\mathfrak{f}_{k}^{ \pm}, k=1, \ldots, n$, is known as a Witt basis for $W^{ \pm}$, respectively. Note that $\left(\mathfrak{f}_{k}^{ \pm}\right)^{2}=0$,

$$
\mathfrak{f}_{j}^{ \pm} \mathfrak{f}_{k}^{ \pm}+\mathfrak{f}_{k}^{ \pm} \mathfrak{f}_{j}^{ \pm}=0
$$

and

$$
\mathfrak{f}_{j}^{+} \mathfrak{f}_{k}^{-}+\mathfrak{f}_{k}^{-} \mathfrak{f}_{j}^{+}=\delta_{j, k} .
$$

These relations correspond to the relations of differential forms $d z_{j}, d \bar{z}_{k}$, and their duals on the alternating algebra $\wedge\left(\mathbb{C}^{n}\right)$. See, for instance $[5,6]$. In fact, $e_{j}=\mathfrak{f}_{j}^{+}+\mathfrak{f}_{j}^{-}$for $j=1, \ldots, n$ and $e_{j}=-i\left(\mathfrak{f}_{j}^{+}-\mathfrak{f}_{j}^{-}\right)$for $j=n+1, \ldots, 2 n$. So our complex Clifford algebra and the alternating algebra are the same.

Following $[2,4]$ we now consider

$$
\frac{1}{2}(I \pm i J) D \text {. }
$$

This splits the Dirac operator, $D$, into a pair of operators $D^{ \pm}$acting over $W^{ \pm}$respectively. So $D=D^{-} \oplus D^{+}$. Explicitly,

$$
D^{+}=\frac{1}{2}\left(\begin{array}{cc}
D & -i D \\
i D & D
\end{array}\right), \quad D^{-}=\frac{1}{2}\left(\begin{array}{cc}
D & i D \\
-i D & D
\end{array}\right) .
$$

Now consider $\mathbb{C}^{n}=\left\{\left(z_{1}, \cdots, z_{n}\right), z_{1}, \cdots, z_{n} \in \mathbb{C}\right\}$.

We have the mappings

$$
P^{ \pm}: \mathbb{C}^{n} \rightarrow W^{ \pm}, \quad\left(z_{1}, \cdots, z_{n}\right) \mapsto z_{1} \mathfrak{f}_{1}^{ \pm}+\cdots+z_{n} \mathfrak{f}_{n}^{ \pm} .
$$


Via these identifications it can be seen that $D^{+}$corresponds to the operator $\bar{\partial}=\sum_{j=1}^{n} d \bar{z}_{j} \frac{\partial}{\partial \bar{z}_{j}}$ and $D^{-}$ corresponds to its dual $\bar{\partial}^{*}$ from several complex variables. See for instance [5, 6]. In this way the Dirac operator corresponds to the operator $\bar{\partial}+\bar{\partial}^{*}$.

\section{Hermitean Clifford Analysis and matrix differential operators}

In [13] the pair of matrix differential operators

$$
\left(\begin{array}{cc}
D^{+} & D^{-} \\
D^{-} & D^{+}
\end{array}\right), \quad\left(\begin{array}{cc}
D^{-} & D^{+} \\
D^{+} & D^{-}
\end{array}\right)
$$

are introduced. Note that

$$
\left(\begin{array}{cc}
D^{+} & D^{-} \\
D^{-} & D^{+}
\end{array}\right)\left(\begin{array}{cc}
D^{-} & D^{+} \\
D^{+} & D^{-}
\end{array}\right)=\left(\begin{array}{cc}
D^{-} & D^{+} \\
D^{+} & D^{-}
\end{array}\right)\left(\begin{array}{cc}
D^{+} & D^{-} \\
D^{-} & D^{+}
\end{array}\right)=\Delta_{2 n}\left(\begin{array}{cc}
I & 0 \\
0 & I
\end{array}\right),
$$

where $\Delta_{2 n}$ is the Laplacian on $\mathbb{R}^{2 n}$. The first matrx operator can be written as

$$
\left(\begin{array}{cc}
D^{+} & D^{-} \\
D^{-} & D^{+}
\end{array}\right)=D^{+}\left(\begin{array}{cc}
I & 0 \\
0 & I
\end{array}\right)+D^{-}\left(\begin{array}{cc}
0 & I \\
I & 0
\end{array}\right)
$$

and the second as

$$
\left(\begin{array}{cc}
D^{-} & D^{+} \\
D^{+} & D^{-}
\end{array}\right)=D^{-}\left(\begin{array}{cc}
I & 0 \\
0 & I
\end{array}\right)+D^{+}\left(\begin{array}{cc}
0 & I \\
I & 0
\end{array}\right) .
$$

Now, the space $\mathbb{R}(2)$ of $2 \times 2$ matrices is a representation of the Clifford algebra $\mathcal{C l}_{2,+}$. See for instances [1]. To see this, consider the basis $1, g_{1}, g_{2}, g_{1} g_{2}$ of $\mathcal{C l}_{2,+}$. So $g_{i}^{2}=1$ for $i=1,2$. Now, make the identification

$$
1 \leftrightarrow\left(\begin{array}{cc}
I & 0 \\
0 & I
\end{array}\right), g_{1} \leftrightarrow\left(\begin{array}{cc}
I & 0 \\
0 & -I
\end{array}\right), g_{2} \leftrightarrow\left(\begin{array}{cc}
0 & I \\
I & 0
\end{array}\right)
$$

So $g_{1} g_{2}$ is identified with $\left(\begin{array}{cc}0 & -I \\ I & 0\end{array}\right)$. This defines an algebra isomorphism between $\mathcal{C l}_{2,+}$ and $\mathbb{R}(2)$.The matrix differential operators now become

$$
D^{+} \otimes 1+D^{-} \otimes g_{2} \sim D^{+}+D^{-} g_{2}, \quad D^{-} \otimes 1+D^{+} \otimes g_{2} \sim D^{-}+D^{+} g_{2},
$$

and

$$
\left(D^{+}+D^{-} g_{2}\right)\left(D^{-}+D^{+} g_{2}\right)=\left(D^{+} D^{-}+D^{-} D^{+}\right)=\Delta_{2 n} .
$$

Further $\mathcal{C l}_{2 n,-} \otimes \mathcal{C l}_{2,+} \cong \mathcal{C} l_{2 n+2,+}$ (see [1] ). So, $D^{+} \otimes 1+D^{-} \otimes g_{2}$ and $D^{-} \otimes 1+D^{+} \otimes g_{2}$ define a couple of Dirac operators defined over a copy of $\mathbb{R}^{2 n}$ lying in $\mathrm{Cl}_{2 n+2,+}$.

The fundamental solution to $D^{+}+D^{-} g_{2} \sim D^{+} \otimes 1+D^{-} \otimes g_{2}$ is, up to a constant,

$$
E_{1}(x)=\frac{x^{-} \otimes 1+x^{+} \otimes g_{2}}{|x|^{2 n}}
$$

where $x=x_{1} e_{1}+\cdots+x_{2 n} e_{2 n}$ and $x^{ \pm}=\frac{1}{2}(I \pm i J) x$.

In matrix form this corresponds to

$$
\frac{1}{|x|^{2 n}}\left(\begin{array}{cc}
x^{-} & x^{+} \\
x^{+} & x^{-}
\end{array}\right)
$$

while the fundamental solution to the operator $D^{-} \otimes 1+D^{+} \otimes g_{2}$ is, up to a constant,

$$
E_{2}(x)=\frac{x^{+} \otimes 1+x^{-} \otimes g_{2}}{|x|^{2 n}} .
$$


In matrix form this gives

$$
\frac{1}{|x|^{2 n}}\left(\begin{array}{ll}
x^{+} & x^{-} \\
x^{-} & x^{+}
\end{array}\right) .
$$

They correspond to the fundamental solutions to the matrix operator given in [3].

The following Borel-Pompeiu and Cauchy integral formulas correspond to the integral formulas appearing in [3].

$$
\begin{aligned}
f(y)= & \frac{1}{\omega_{n}} \int_{S} E_{+}(x)\left(n^{+}(x) \otimes 1+n^{-}(x) \otimes g_{2}\right) f(x) d \sigma(x) \\
+ & \frac{1}{\omega_{n}} \iint_{\Omega} E_{+}(x)\left(D^{+} \otimes 1+D^{-} \otimes g_{2}\right) f(x) d \mu(x) \\
= & \frac{1}{\omega_{n}} \int_{S} E_{+}(x)\left(n^{+}(x)+n^{-}(x) g_{2}\right) f(x) d \sigma(x) \\
& +\frac{1}{\omega_{n}} \iint_{\Omega} E_{+}(x)\left(D^{+}+D^{-} g_{2}\right) f(x) d \mu(x),
\end{aligned}
$$

and if $\left(D^{+} \otimes 1+D^{-} \otimes g_{2}\right) f(x)=0$ this integral gives a Cauchy integral formula.

There are similar integral formulas obtained by replacing $E_{+}$by $E_{-}, n^{+}(x) \otimes 1+n^{-}(x) \otimes g_{2}$ by $n^{-}(x) \otimes$ $1+n^{+}(x) \otimes g_{2}$ and $D^{+} \otimes 1+D^{-} \otimes g_{2}$ by $D^{-} \otimes 1+D^{+} \otimes g_{2}$.

\section{Conformal transformations in the Hermitean Case}

In [14] and elsewhere it is shown that the Euclidean Dirac operator is invariant under conformal transformations. A theorem of Liouville tells us that for dimensions 3 and above the only conformal transformations are Möbius transformations indentified with $S O(2 n+1,1)$. These are transformations generated from translations, dilations, orthogonal transformations and the Kelvin inversion $\left(x \rightarrow \frac{x}{|x|^{2}}\right)$. The question now is what subgroup of the conformal group preserves $D^{+}$or equivalently, $\bar{\partial}$ and $\bar{\partial}^{*}$ ? As $D^{ \pm}$are both homogeneous and with constant coefficients they are invariant under both translations and dilations.

As for orthogonal transformations, such a transformation must preserve the spaces $W^{ \pm}$. So we need to restrict to transformations in $S O(2 n)$ that commute with $J$. It is known that this is a subgroup of $S O(2 n)$ isomorphic to $\mathcal{U}(n)$.

To proceed further we need the spin group. First, consider

$$
\left\{a \in \mathcal{C}_{2 n,-}: a=y_{1} \cdots y_{p}, \text { for } y_{1}, \cdots, y_{p} \in \mathbb{S}^{2 n-1}\right\} .
$$

This set is a group under Clifford algebra multiplication. It is the pin group and it is denoted by $\operatorname{Pin}(2 n)$. If we restrict so that $p$ is even we obtain a subgroup called the spin group. It is denoted by $\operatorname{Spin}(2 n)$.

For $a=y_{1} \cdots y_{2 p} \in \operatorname{Spin}(2 n)$ we denote $y_{2 p} \cdots y_{1}$ by $\tilde{a}$.

It is well known, [12] for instance, that for $x \in \mathbb{R}^{2 n}$, axa defines a special orthogonal transformation on $\mathbb{R}^{2 n}$. In fact, [12], there is a surjective group homomorphism

$$
\theta: \operatorname{Spin}(2 n) \rightarrow S O(2 n), \quad a \mapsto \theta_{a},
$$

where $\theta_{a} x:=a x \tilde{a}$. The kernel of this homomorphism is $\{ \pm 1\},[12]$.

We have previously noted that the operators $D^{ \pm}$are invariant under actions of a copy of $\mathcal{U}(n) \subset S O(2 n)$. It follows that this copy of $\mathcal{U}(n)$ has a double covering, $\mathcal{U}^{\prime}(n) \subset \operatorname{Spin}(2 n)$, also isomorphic to $\mathcal{U}(n)$. Further, 
following [8] then if $\Omega$ is a domain in $\mathbb{R}^{2 n}$ and $y=a x \tilde{a} \in \Omega$, with $a \in \mathcal{U}^{\prime}(n)$ then if

$$
D^{ \pm} f(y)=0 \quad \text { then } \quad D^{ \pm} \tilde{a} f(a x \tilde{a})=0,
$$

where $D^{ \pm}$now acts with respect to the variable $x$.

This situation differs from what is considered in several variables for the operators $\bar{\partial}$ and $\bar{\partial}^{*}$ in $\mathbb{C}^{n}$. There these operators are considered as acting strictly on $(p, q)$ forms, see for instance [5]. The action of $\tilde{a}$ on the function $f(a x \tilde{a})$ is a spherical action that does not preserve $(p, q)$ forms. It preserves the spinor subspaces of the algebra $\mathrm{Cl}_{2 n,-}$ or $\mathrm{Cl}_{2 n}(\mathbb{C})$, see [12].

We now turn to consider the case of the Kelvin inversion. Given a hypersurface $S$ in $\mathbb{R}^{2 n}$ then under Kelvin inversion the surface element $n(y) d \sigma(y)$ is transformed to $G(x) n(x) G(x) d \sigma(x)$, where $y \in S, n(y)$ is the outer pointing unit vector perpendicular to the tangent space $T S_{y}$, and $\sigma$ is the Lebesgue measure on $S$. We want to know if the spaces $W^{ \pm}$are preserved under the Kelvin inversion and in particular if the operators $D^{ \pm}$are also preserved. As $G(x)=\frac{x}{|x|^{2 n}}$, this boils down to determine whether or not $J$ commutes with the action $x n(x) x$ of $x$ on $n(x)$. Note that $x n(x) x$ describes (up to the sign) a reflection of $n(x)$ in the direction of $x$. So $x n(x) x$ is a vector in $\mathbb{R}^{2 n}$.

As $J$ is a matrix in $S O(2 n)$ and the vector $x n(x) x$ is defined in terms of Clifford multiplication it is better to make the notation uniform. The action of $J$ on $\mathbb{R}^{2 n}$ gives a counterclockwise rotation of $\pi / 2$ in each plane spanned by $e_{i}, e_{i+n}$ for $i=1, \cdots n$. So a lifting of $J$ to $\operatorname{Spin}(2 n)$ gives

$$
\pm j= \pm \frac{1}{\sqrt{2}}\left(1+e_{1} e_{1+n}\right) \cdots \frac{1}{\sqrt{2}}\left(1+e_{n} e_{2 n}\right)= \pm \frac{1}{2^{n / 2}}\left(1+e_{1} e_{1+n}\right) \cdots\left(1+e_{n} e_{2 n}\right) .
$$

It should be noted that each pair $\left(1+e_{i} e_{i+n}\right)\left(1+e_{s} e_{s+n}\right)$ commutes with each other. It follows that it is enough to compare the terms

$$
\left(1+e_{1} e_{1+n}\right) x n(x) x\left(1+e_{1+n} e_{1}\right)
$$

and

$$
x\left(1+e_{1} e_{1+n}\right) n(x)\left(1+e_{1+n} e_{1}\right) x .
$$

In fact, it is enough to compare the subterms $\left(1+e_{1} e_{1+n}\right) x n(x) x$ and $x\left(1+e_{1} e_{1+n}\right) n(x) x$. As the hypersurface $S$ is arbitrary we can place $x=e_{1}$. In this case the terms

$$
\left(1+e_{1} e_{1+n}\right) e_{1}=\left(e_{1}+e_{1+n}\right) \text { and } e_{1}\left(1+e_{1} e_{1+n}\right)=\left(e_{1}-e_{1+n}\right)
$$

differ by a sign. It follows that the operators $D^{ \pm}$are not invariant under the Kelvin transformation. Consequently the operators $\bar{\partial}$ and $\bar{\partial}^{*}$ are not invariant under the Kelvin transformation.

Summing up we have established:

Theorem 4.1. The operators $D^{ \pm}$(and consequently, $\bar{\partial}$ and $\bar{\partial}^{*}$ ) are only invariant under translations, dilations and actions of the group $\mathcal{U}^{\prime}(n) \subset \operatorname{Spin}(2 n)$.

\section{Kohn Dirac and Laplacian operators in $S^{2 n-1}$}

In the previous sections we have shown that with the exception of the action of $\mathcal{U}^{\prime}(n)$ on $D^{ \pm},\left(\bar{\partial}, \bar{\partial}^{*}\right)$, much of the Hermitian Clifford analysis already exists in several complex variables over $\mathbb{C}^{n}$. This leads to the question of trying to find ways in which to develop Hermitian Clifford analysis that are more meaningful and original.

One starting point is to look at its analogue over odd dimensional spheres. 
Consider $\mathbb{S}^{2 n-1} \subset \mathbb{C}^{n}$. Consider also $x \in \mathbb{S}^{2 n-1}$ and $T \mathbb{S}_{x}^{2 n-1} \subset \mathbb{C}^{n}$. In several complex variables [5,6] one considers the complex subspace $L_{x}$ of $T \mathbb{S}_{x}^{2 n-1}$ where

$$
L_{x}=\left\{y \in T \mathbb{S}_{x}^{2 n-1}: i y \in T \mathbb{S}_{x}^{2 n-1}\right\} .
$$

Here, $n \geq 2$. This gives rise to a situation that does not occur in one complex variable. We now have a complex bundle $\mathbf{L} \subset T \mathbb{S}^{2 n-1}$ where each fiber is the complex space $L_{x}$. Consider now the canonical projection

$$
P_{x}: T \mathbb{S}_{x}^{2 n-1} \rightarrow L_{x}
$$

This gives rise to the projection

$$
\mathbf{P}: T \mathbb{S}_{x}^{2 n-1} \rightarrow \mathbf{L} .
$$

Now consider a hypersurface $S^{\prime}$ bounding a domain $\Omega^{\prime}$ in $\mathbb{S}^{2 n-1}$. If we consider $S^{\prime}$ to be sufficiently smooth we can consider smooth $\mathcal{C}_{2 n}(\mathbb{C})$-valued functions defined in a neighbourhood of $\Omega^{\prime}$ within $\mathbb{S}^{2 n-1}$ and following [10] we have the following version of the Stokes' Theorem.

\section{Theorem 5.1.}

$$
\int_{S^{\prime}} f(x) n(x) g(x) d \sigma(x)=\iint_{\Omega^{\prime}}\left(\left(f(x) D_{s}\right) g(x)+f(x)\left(D_{s} g(x)\right)\right) d x,
$$

where $n(x)$ is the outward pointing unit vector in $T S_{x}^{\prime}$. Further $D_{s}$ is the spherical Dirac operator $x\left(\Gamma_{x}+\frac{n}{2}\right)$, where $\Gamma_{x}:=\sum_{1 \leq i<j \leq 2 n-1} e_{i} e_{j}\left(x_{i} \partial_{x_{j}}-x_{j} \partial_{x_{i}}\right)$.

We may also consider the modified integral

$$
\int_{S^{\prime}} f(x) P_{x}(n(x)) g(x) d \sigma(x) .
$$

Applying the Stokes' Theorem to this integral gives the integral

$$
\iint_{\Omega^{\prime}}\left(\left(f(x)\left(D_{s} P_{x}\right)\right) g(x)+f(x)\left(\left(P_{x} D_{s}\right) g(x)\right)\right) d x .
$$

For each $x \in \mathbb{S}^{2 n-1}$, we have $i x \in T \mathbb{S}_{x}^{2 n-1}$, so $i x \notin L_{x}$. Consequently, $P_{x}(i x)=0$. Therefore, $P_{x}\left(D_{s}\right)=$ $P_{x}\left(x \Gamma_{x}\right)$. It follows that this operator is an isomorphic copy of the sub-elliptic Dirac operator $\bar{\partial}_{b}+\bar{\partial}_{b}^{*}$ over $S^{2 n-1}$. This Dirac operator is also known as the Kohn Dirac operator (see [11]). Therefore, $\left(P_{x}\left(x \Gamma_{x}\right)\right)^{2}=\square_{b}$, the Kohn Laplacian. The operators $\partial_{b}, \bar{\partial}_{b}^{*}$ and $\square_{b}$ are all defined in $[5,6]$ and elsewhere.

The group of conformal transformations that preserves the unit $\mathrm{b}$ all $B(0,1) \subset \mathbb{R}^{2 n}$, and its boundary, is $S O(2 n, 1)$. However, we have identified $\mathbb{R}^{2 n}$ with $\mathbb{C}^{n}$. We need to restrict to the subgroup of $S O(2 n, 1)$ that preserves the Hermitian structure of $\mathbb{C}^{n}$. At this point we need to reference or show that this group is $\mathcal{U}(n, 1)$. We also need to show that the invariant group for $P_{x}\left(D_{s}\right)$ is the double cover of $\mathcal{U}(n, 1)$ within $\operatorname{Spin}(2 n)$. A start is the following: note that if $\left(f(x)\left(D_{s} P_{x}\right)\right)=\left(P_{x} D_{s}\right) g(x)=0$ then we have the following version of the Cauchy's Theorem:

\section{Theorem 5.2.}

$$
\int_{S^{\prime}} f(x) P_{x}(n(x)) g(x) d \sigma(x)=0 .
$$

By allowing $S^{\prime}$ to vary it follows that the group of diffeomorphisms that preserves the Kohn-Dirac operator $P_{x}\left(D_{s}\right)$ is a subgroup of $\operatorname{Spin}(2 n, 1)$ whose projection preserves the bundle $\mathbf{L}$.

This should be $\mathcal{U}(n, 1)$, with a double cover in $\operatorname{Spin}(2 n, 1)$ isomorphic to $\mathcal{U}(n, 1)$. 


\section{Realization ON $\mathbb{S}^{3}$}

To make the above points more clear we consider the three-dimensional sphere as a special example. Furthermore, we will consider for the moment quaternionic valued functions.

Let us introduce the following first-order differential operators

$$
\begin{aligned}
X_{\mathbf{i}} & =x_{0} \partial_{x_{1}}-x_{1} \partial_{x_{0}}+x_{3} \partial_{x_{2}}-x_{2} \partial_{x_{3}}, \\
X_{\mathbf{j}} & =x_{0} \partial_{x_{2}}-x_{2} \partial_{x_{0}}+x_{1} \partial_{x_{3}}-x_{3} \partial_{x_{1}}, \\
X_{\mathbf{k}} & =x_{0} \partial_{x_{3}}-x_{3} \partial_{x_{0}}+x_{1} \partial_{x_{2}}-x_{2} \partial_{x_{1}}
\end{aligned}
$$

These differential operators are skew-symmetric with respect to the Riemannian surface form $d S$ on $\mathbb{S}^{3}$ given by

$$
d S=\sum_{j=0}^{3}(-1)^{j} x_{j} d \hat{x}_{j}
$$

where $d \hat{x}_{j}$ is generated from the oriented volume form $d x$ with $d x_{j}$ being omitted.

The sub-Laplacian $\square_{b}$ is given by

$$
\square_{b}=-X_{\mathbf{i}}^{2}-X_{\mathbf{k}}^{2}
$$

while the Laplacian is given by

$$
\Delta=-X_{\mathbf{i}}^{2}-X_{\mathbf{j}}^{2}-X_{\mathbf{k}}^{2}
$$

Moreover, we have the sub-Dirac operator

$$
D_{b}:=\mathbf{i} X_{\mathbf{i}}+\mathbf{k} X_{\mathbf{k}}
$$

Keep in mind that in general its square is not the sub-Laplacian since $X_{\mathbf{j}}=\left[X_{\mathbf{i}}, X_{\mathbf{k}}\right]$.

We can identify $\mathbb{R}^{4} \sim \mathbb{H}$ with $\mathbb{C}^{2}$ in the usual way via $z=z_{1}+\mathbf{i} z_{2}$, with $z_{1}, z_{2} \in \mathbb{C}_{\mathbf{j}}:=\{a+b \mathbf{j}, a, b \in \mathbb{R}\}$. The one-parameter transformation group generated by $X_{\mathbf{j}}$ corresponds to the complex multiplication with $\lambda=(a+b \mathbf{j})$ from the right. The resulting orbits on $\mathbb{S}^{3}$ span the complex projective space $P^{1} \mathbb{C}$ and we get the Hopf bundle

$$
\pi_{R}: \mathbb{S}^{3} \mapsto P^{1} \mathbb{C}
$$

The complex line bundle $L^{l}$ on $P^{1} \mathbb{C}$ is associated to the character $\chi_{l}(\lambda)=\lambda^{l}$.

As usual we can now consider the space $\Pi_{N}$ of homogeneous polynomials of degree $N$ in the variables $x_{0}, x_{1}, x_{2}, x_{3}$. We denote by $H_{N}$ the subspace of harmonic polynomials in $\Pi_{N}$. For this subspace we have the classic Fischer decomposition

$$
\Pi_{N}=H_{N}+\left(\sum_{i=0}^{3} x_{i}^{2}\right) \Pi_{N-2} .
$$

The space $H_{N}$ restricted to the sphere $\mathbb{S}^{3}$ is the eigenspace $\mathbf{H}_{N}$ of the Laplacian $\Delta$ with respect to the eigenvalue $\lambda_{N}=N(N+2)$ with dimension $(N+1)^{2}$.

Let us now consider the subspace $\Pi_{n, m}, n+m=N$, of $\Pi_{N}$ defined by

$$
\Pi_{n, m}=\left\{p \in \Pi_{N}: p\left(z_{0} e^{i t}, z_{1} e^{i t}, \overline{z_{0} e^{i t}}, \overline{z_{1} e^{i t}}\right)=e^{i(n-m) t} p\left(z_{0}, z_{1}, \overline{z_{0}}, \overline{z_{1}}\right)\right\}
$$

and the subspace $H_{n, m}=H_{N} \cap \Pi_{n, m}$. In this subspace we have $X_{\mathbf{j}} p=(n-m) p$. This leads to the decomposition

$$
H_{N}=\sum_{n+m=N, n, m \geq 0} \oplus H_{n, m}
$$


Furthermore, we have harmonic Fischer decomposition

$$
\Pi_{n, m}=H_{n, m}+\left(\left|w_{0}\right|^{2}+\left|w_{1}\right|^{2}\right) \Pi_{n-1, m-1} .
$$

Moreover, each of the eigenspaces $H_{n, m}$ can be decomposed in terms of eigenspaces of the Dirac operator,

$$
H_{n, m}=M_{n, m}+\left(w_{0}+w_{1}\right) M_{n-1, m}+\left(\bar{w}_{0}+\bar{w}_{1}\right) M_{n, m-1}+w_{0} \bar{w}_{1} M_{n-1, m-1}+\bar{w}_{0} w_{1} M_{n-1, m-1},
$$

where $M_{k, s}=\left\{p \in \Pi_{k, s}: D p=0\right\}$.

Furthermore, for any $p \in H_{n, m}$ we have

$$
\Delta p=-\left(X_{\mathbf{i}}^{2}+X_{\mathbf{k}}^{2}\right) p+(n-m)^{2} p=\square_{b} p+(n-m)^{2} p
$$

as well as

$$
D_{b} p=\left(\mathbf{i} X_{\mathbf{i}}+\mathbf{k} X_{\mathbf{k}}\right) p+\mathbf{j}(n-m) p
$$

and we denote the restriction of $H_{n, m}$ to $\mathbb{S}^{3}$ by $\mathbf{H}_{n, m}$ as well as the restriction of $M_{n, m}$ to $\mathbb{S}^{3}$ by $\mathbf{M}_{n, m}$.

This leads to the following lemma.

Lemma 6.1. The space $\mathbf{M}_{n, m}$ is the eigenspace of the sub-Dirac operator $D_{b}$ with respect to the eigenvalue

$$
-N-\mathbf{j} l=-(1+\mathbf{j}) n-(1-\mathbf{j}) m
$$

with multiplicity $N+2$. Here, $l=n-m, N=n+m, n, m \geq 0$. Furthermore, the space $\mathbf{H}_{n, m}$ is the eigenspace of the sub-Laplacian $\square_{b}$ with respect to the eigenvalue

$$
N(N+2)-l^{2}=4 m^{2}+4 m(1+|l|)+2|l|
$$

with multiplicity $|l|+2 m+1$.

Let us consider now the subspace $F^{l}$ of $C^{\infty}\left(\mathbb{S}^{3}\right)$ of homogeneous sections on the complex line bundle $L^{l}$, i.e. $F^{l} \sim \Gamma\left(L^{l}\right)$ and

$$
F^{l}=\left\{f \in C^{\infty}\left(\mathbb{S}^{3}\right): f(x(a+b \mathbf{j}))=(a+b i)^{-l} f(x), a, b \in \mathbb{R}, a^{2}+b^{2}=1\right\} .
$$

The sub-Laplacian can be identified with the horizontal Laplacian

$$
\square_{b}: \Gamma\left(L^{l}\right) \mapsto \Gamma\left(L^{l}\right)
$$

This identification allows us to decompose $F^{l}$ as

$$
F^{l} \sim \Gamma\left(L^{l}\right)=\sum_{n-m=l, n, m \geq l} \oplus H_{n, m}
$$

into eigenspaces of the horizontal Laplacian.

The sub-Dirac operator can be identified with the horizontal Dirac operator

$$
D_{b}: \Gamma\left(L^{l}\right) \mapsto \Gamma\left(L^{l}\right)
$$

This identification allows us to decompose $F^{l}$ as

$$
F^{l} \sim \Gamma\left(L^{l}\right)=\sum_{n-m=l, n, m \geq l} \oplus M_{n, m}
$$

into eigenspaces with respect to the horizontal Dirac operator. 


\section{Subelliptic Dirac operator}

Following [7, 9] it is desirable to ask what manifolds besides $\mathbb{S}^{2 n-1}$ admit a subelliptic Dirac operator. Clearly it should be a CR manifold with some sort of spin structure.

In $\mathbb{C}^{n}$ we saw an invariance under a subgroup of $\operatorname{Spin}(2 n)$ isomorphic to $\mathcal{U}(n)$. This suggests that we are locking for a CR manifold with principle bundle whose fibers are isomorphic to $\mathcal{U}(n)$ and with a global double cover also with fiber isomorphic to $\mathcal{U}(n)$.

\section{Irreducible RePresentations in $\mathcal{U}^{\prime}(n)$}

Within the Clifford algebras $C \ell_{m}$ the irreducible representation spaces for $\operatorname{Spin}(m)$ are the spinor spaces. Here though we are dealing with the subgroup $\mathcal{U}^{\prime}(n)$ of $\operatorname{Spin}(2 n)$.

As point out in [15] the spinor spaces are no longer irreducible subspaces of $\mathcal{U}^{\prime}(n)$. Furthermore, in several complex variables one is interested specifically in $(p, q)$ sections. If $\underline{z}=a \underline{\omega} \tilde{a}$ and $f(\underline{z})$ is the isomorphic equivalent of a $(p, q)$ form in the context we consider here then in general $\tilde{a} f(a \underline{\omega} \tilde{a})$ is not the equivalent of a $(p, q)$ form.

To overcome this instead of considering $\tilde{a} f(a \underline{\omega} \tilde{a})$ we consider $\tilde{a} f(a \underline{\omega} \tilde{a}) a$. Note that first $\tilde{a} f_{j}^{ \pm} a$ remains in $W^{ \pm}$, for $j=1, \cdots, n$ and $a \in \mathcal{U}^{\prime}(n)$. Note further that $\tilde{a} a=1$ for each $a \in \mathcal{U}^{\prime}(n)$, or for that matter $\operatorname{Spin}(2 n)$.

Consequently given $f_{j_{1}}^{+} \cdots f_{j_{r}}^{+} f_{k_{1}}^{-} \cdots f_{k_{s}}^{-}$then

$$
\tilde{a}\left(f_{j_{1}}^{+} \cdots f_{j_{r}}^{+} f_{k_{1}}^{-} \cdots f_{k_{s}}^{-}\right) a=\tilde{a} f_{j_{1}}^{+} a \tilde{a} f_{j_{2}}^{+} a \cdots \tilde{a} f_{j_{r}}^{+} a \tilde{a} f_{k_{1}}^{-} a \cdots \tilde{a} f_{k_{s}}^{-} a .
$$

It follows that if $f(\underline{z})$ is the equivalent of a $(p, q)$ form in the context described here then so is $\tilde{a} f(a \underline{\omega} \tilde{a}) a$.

\section{REFERENCES}

[1] M. F. Atiyah, R. Bott, and A. Shapiro, Clifford Modules, Topology 3 (Suppl. 1) (1964), 3-38.

[2] F. Brackx, H. De Schepper, F. Sommen The Hermitian Clifford Analysis Toolbox, Adv. Appl. Clifford Alg. 18 (2008), 451-487.

[3] F. Brackx, B. De Knock, H. De Schepper, F. Sommen, On Cauchy and Martinelli-Bochner integral formulae in Hermitian Clifford analysis, Bull. Braz. Math. Soc. (N.S.) 40 (3) (2009) 395416.

[4] F Brackx, H De Schepper, David Eelbode, and V Soucek, The Howe dual pair in Hermitean Clifford analysis, Rev. Mat. Iberoamericana, 26(2), (2010), 449-479.

[5] A. Boggess, CR Manifolds and the Tangential Cauchy Riemann Complex, Studies in Advanced Mathematics, CRC Press, 1991.

[6] J. P. D'Angelo, Several Complex Variables and the Geometry of Real Hypersurfaces, CRC Press, 1993.

[7] C. L. Epstein, Subelliptic Spin $\mathbb{C}$ Dirac operators, I, Annals of Math., 166, (2007), 183-214.

[8] M. G. Eastwood, J. Ryan, Aspects of Dirac operators in analysis, Milan J. Math., 75, (2007), 91-116.

[9] I. Kath, O. Ungermann, Spectra of sub-Dirac operators on certain nilmanifolds, Math. Scand., 117 (1), (2015).

Available at: http://arxiv.org/abs/1311.2418

[10] H. Liu and J. Ryan, Clifford analysis techniques for spherical pde, J. Fourier Anal. Appl., 8 (6) (2002), $535-564$.

[11] R. Petit, Spin ${ }^{\text {- }}$-structures and Dirac operators on contact manifolds, Differ. Geom. Appl., 22 (2005), $229-252$.

[12] I. Porteous, Clifford Algebras and the Classical Groups, Cambridge University Press, 1995.

[13] R. Rocha-Chávez, M. Shapiro, F. Sommen, Integral Theorems for Functions and Differential Forms in $\mathbb{C}^{m}$, Res. Notes Math., vol. 428, Chapman \& Hall/CRC, Boca Raton, FL, 2002.

[14] J. Ryan, Conformally covariant operators in Clifford analysis, Z. Anal. Anwend., 14, (1995), 677-704.

[15] S. Shirrell, R. Walter, Hermitian Clifford Analysis and Its Connections with Representation Theory, submitted.

Acknowledgment. The work of the first and second authors was partially supported by Portuguese funds through the CIDMA - Center for Research and Development in Mathematics and Applications, and the 
Portuguese Foundation for Science and Technology ("FCT-Fundação para a Ciência e a Tecnologia"), within project UID/MAT/ 0416/2013.

Cidma, Departamento de Matemática, Universidade de Aveiro, Campus de Santiago, P-3810-193, Aveiro, PorTUGAL

E-mail address: pceres@ua.pt

Cidma, Departamento de Matemática, Universidade de Aveiro, Campus de Santiago, P-3810-193, Aveiro, PorTUGAL

E-mail address: ukaehler@ua.pt

Department of Mathematical Sciences, University of Arkansas, Fayetteville, AR 72701, U.S.A.

E-mail address: jryan@uark.edu 